\title{
Parvalbumin Interneuron Plasticity for Consolidation of Reinforced Learning
}

\author{
Matteo Tripodi, Komal Bhandari, Ananya Chowdhury, ${ }^{1}$ Arghya Mukherjee, \\ AND PICO CARONI \\ Friedrich Miescher Institute for Biomedical Research, 4058 Basel, Switzerland \\ Correspondence: caroni@fmi.ch
}

\begin{abstract}
Parvalbumin (PV) basket cells are widespread local interneurons that inhibit principal neurons and each other through perisomatic boutons. They enhance network function and regulate local ensemble activities, particularly in the $\gamma$ range. Organized network activity is critically important for long-term memory consolidation during a late time window $11-15 \mathrm{~h}$ after acquisition. Here, we discuss the role of learning-related plasticity in PV neurons for long-term memory consolidation. The plasticity can lead to enhanced (high-PV) or reduced (low-PV) expression of PV/GAD67. High-PV plasticity is induced upon definite reinforced learning in early-born PV basket cells, whereas low-PV plasticity is induced upon provisional reinforced learning in late-born PV basket cells. The plasticity is first detectable $6 \mathrm{~h}$ after acquisition, at the end of a time window for memory specification through experience, and is critically important $11-15 \mathrm{~h}$ after acquisition for enhanced network activity and longterm memory consolidation. High- and low-PV plasticity appear to regulate activity in distinct local networks of principal neurons and PV basket cells. These findings suggest how flexibility and stability in learning and memory might be implemented through parallel circuits and networks.
\end{abstract}

Parvalbumin (PV) basket cells are widely distributed and abundant GABAergic inhibitory interneurons that provide powerful local feedforward and feedback inhibition onto principal excitatory neurons (Bartos et al. 2007; $\mathrm{Hu}$ et al. 2014). In addition, PV basket cells inhibit each other reciprocally through perisomatic innervation and are dynamically coupled electrically through gap junctions (Bartos et al. 2007; Hu et al. 2014). PV basket cells filter activation of principal neurons, and networks of PV basket cells have major roles in regulating local ensemble activities, including $\theta$ and $\gamma$ oscillations (Fuchs et al. 2007; Cardin et al. 2009; Sohal et al. 2009; Kuhlman et al. 2010; Isaacson and Scanziani 2011; Buzsáki and Wang 2012; Lee et al. 2012; Hu et al. 2014). Synaptic regulation of PV basket cells has been implicated in adult learning, and the maturation state of PV basket cells has been implicated in critical period-type plasticity (Hensch et al. 1998; Hensch 2005; Di Cristo et al. 2007; Southwell et al. 2010; Kuhlman et al. 2013). We recently discovered that PV basket cells show robust plasticity upon reinforced forms of learning in the adult (Donato et al. 2013), and that two subpopulations of PV basket cells generated during the first/second half of neurogenesis in the median ganglionic eminence show plasticity upon definite (early-born)/provisional (late-born) learning (Donato et al. 2015). PV neuron plasticity only becomes detectable with a delay of $\sim 6 \mathrm{~h}$ after the initial learning event, and persists for 2-3 d (Donato et al. 2015; Karunakaran et al. 2016). In this review, we focus on the function of this unusual form of cellular plasticity and on what it reveals about circuit and systems mechanisms of long-term memory consolidation.

Analysis of learning-related plasticity markers such as pERK, cFos, and Arc expression in the hippocampus after fear conditioning revealed a second peak of expression from +9 to $+15 \mathrm{~h}$ after acquisition (Trifilieff et al. 2006, 2007; Bekinschtein et al. 2007; Katche et al. 2010, 2013; Caroni et al. 2014; Karunakaran et al. 2016). Interfering with dopamine D1 receptor (D1R) signaling or organized network activity at $+12 \mathrm{~h}$ after fear conditioning suppressed long-term consolidation of fear memory, providing evidence for the existence of a late time window at +12 h critically important for long-term memory consolidation (Girardeau et al. 2009, 2014; Rossato et al. 2009; Carr et al. 2011; Jadhav et al. 2012; McNamara et al. 2014). As discussed below, learning-related PV neuron plasticity appears to be specifically important to support offline network activity essential for long-term memory consolidation upon reinforced learning during the late time window at $+12-15 \mathrm{~h}$.

\section{PV NEURON PLASTICITY OF OPPOSITE SIGNS UPON PROVISIONAL AND DEFINITE REINFORCED LEARNING}

Reinforced trial-and-error learning tasks provide attractive experimental paradigms to investigate how plasticity is flexibly adjusted during learning. Thus, effective acqui-

\footnotetext{
${ }^{1}$ Present address: Departments of Neurobiology, Psychology, Psychiatry, and Biobehavioral Sciences, Integrative Center for Learning and Memory, Brain Research Institute, UCLA, Los Angeles, California 90095, USA

(C) 2018 Tripodi et al. This article is distributed under the terms of the Creative Commons Attribution-NonCommercial License, which permits reuse and redistribution, except for commercial purposes, provided that the original author and source are credited.
} 
sition and flexible combination of potentially task-relevant information is essential during early phases of trialand-error learning, whereas reliable application of validated routines dominates toward learning curve completion (Kaelbling et al. 1996). We found that at the circuit level, these contrasting requirements are correlated to shifts in the configuration of local PV-expressing basket cell networks, which show pronounced plasticity induced by certain forms of learning (Fig. 1; Donato et al. 2013). Thus, PV network configurations enriched in neurons expressing low levels of PV and GAD67 and showing relatively high inhibitory connectivity onto them are induced locally early during trial-and-error incremental learning, when they are required for effective learning (Fig. 1A-E). Such "low-PV configurations" promote acquisition and retention of new memories and structural synaptic plasticity (Donato et al. 2013). In contrast, configurations enriched in neurons expressing high levels of PV and GAD67 and showing high excitatory connectivity onto them are induced upon Pavlovian conditioning and toward completion of trial-and-error learning protocols. Such "high-PV configurations" interfere with the acquisition of new incidental memories (Familiar Object Recognition test) and structural synaptic plasticity (Fig. 1D; Donato et al. 2013). The PV plasticity was not detected in tasks involving memory formation in the absence of reinforce-
A
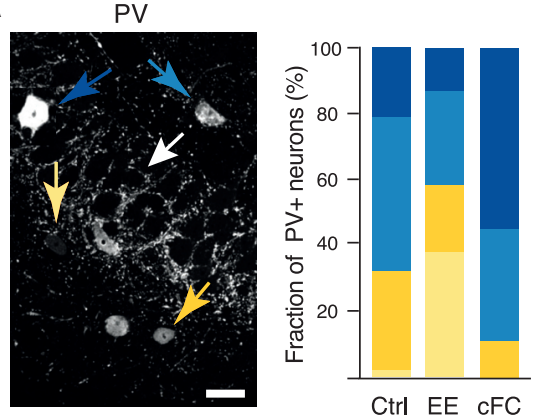

C

Excitation/inhibition versus PV



E

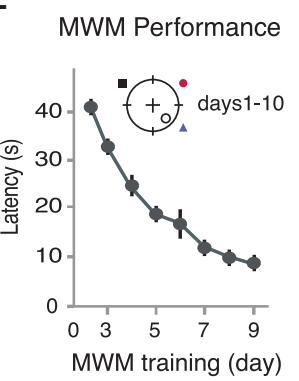

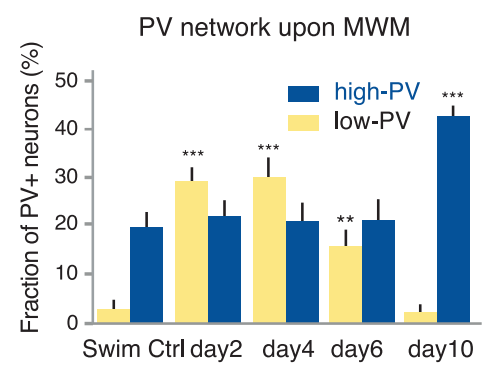

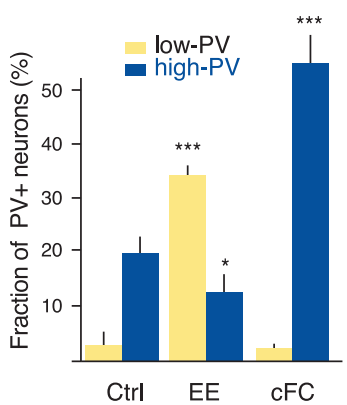

PV-Pharmacogenetic in dCA3:

PV network configuration and hippocampal memory (FOR)
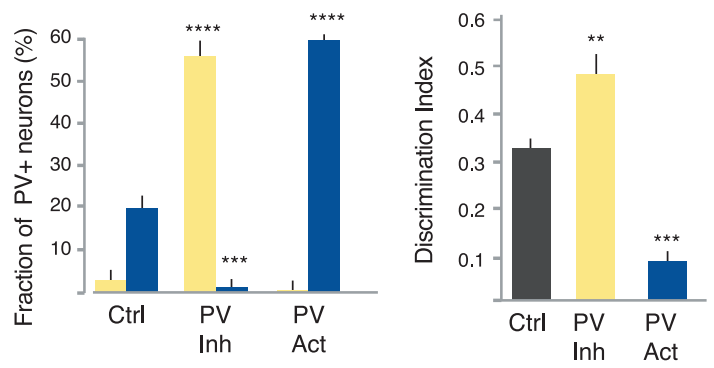

$\mathbf{F}$

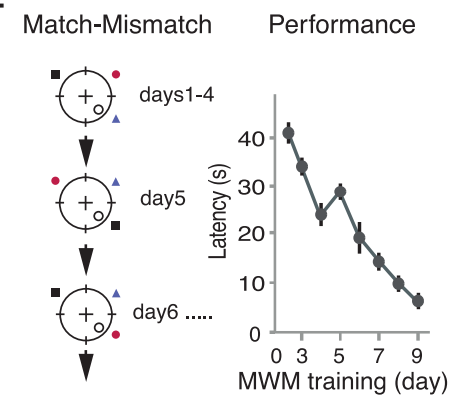

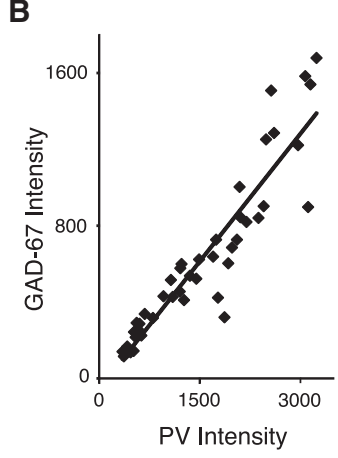

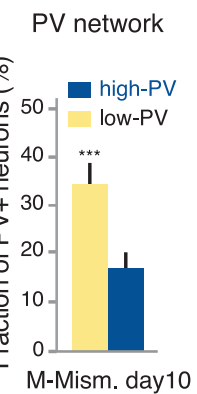

Figure 1. Learning-induced plasticity of PV basket cells. (A) Experience-related plasticity of PV neurons. (Arrows) High- (dark blue), intermediate high- (blue), intermediate low- (gold), and low-PV (yellow) neurons. Relative contents of PV neurons in control (Ctrl), enriched (EE, $3 \mathrm{wk}$ ), and fear-conditioned (cFC, day 1) mice. (B) PV/GAD67 signal relationship in individual PV neurons from Ctrl, EE, and $\mathrm{cFC}$ mice. $(C)$ Local densities of excitatory and inhibitory synaptic puncta as a function of PV immunoreactivity signal. $(D)$ PV neuron plasticity induced upon pharmacogenetic activation or inhibition of dCA3 PV neurons (values $31 \mathrm{~h}$ after ligand delivery) and impact on hippocampal memory. (E) Morris water maze learning curve (left) and PV network configuration in CA3b during MWM learning $(r i g h t)$. $(F)$ Persistence of low-PV configuration in spite of spatial learning when external cues are rearranged daily, from training day 5 on. Schematic of match-mismatch protocol (left), corresponding learning curve (center), and PV network configuration on day 10 for match-mismatch protocol (right). (Adapted from Donato et al. 2013.) 
ment, suggesting that it might be specifically induced upon reinforced learning. Furthermore, the plasticity was specifically detected in brain areas known to have a role in the particular form of learning (e.g., hippocampus for the water maze but not for a motor skill task such as staying on an accelerating rotating rod, and primary motor cortex but not hippocampus for the motor skill task).

Two additional observations warrant mentioning here. First, when a variation of the standard water maze protocol was introduced so that, from training day 5 on, mice had to adjust each day to a new position of the hidden platform relative to allocentric cues on the surrounding walls, mice rapidly adjusted to this more challenging protocol, but the high-PV plasticity consistently observed once mice had reliably learned the spatial map did not occur (Fig. 1F; Donato et al. 2013). This observation suggests that a shift to a high-PV network configuration might specifically be induced when mice effectively manage a task in a way that does not anymore involve unexpected features (such as the relationship between allocentric cues and the position of the hidden platform). Rather than reflecting performance, high-PV plasticity might therefore reflect the absence of deviations and unexpected value-related findings in a trialand-error task. The second informative observation is that the learning-related plasticity does not seem to induce any distinct new phenotype within the population of local PV neurons. Instead, the plasticity appears to shift the relative prevalence of PV neurons with a given distribution of PV/ GAD67 expression of excitatory versus inhibitory synaptic puncta onto them (Donato et al. 2013, 2015). This suggests the presence of consistent, predictable and reversible learning-induced shifts in the relative prevalence of local PV neurons with relatively high- or low-PV/GAD67 expression levels. Given the high degree of connectivity between individual local PV neurons and principal neurons and among the PV neurons themselves, these observations suggest that the plasticity induced upon certain types and stages of learning might influence the functioning of local principal and inhibitory neuron networks.

\section{DISTINCT PV NEURON SUBPOPULATIONS IMPLEMENT PLASTICITY UPON PROVISIONAL AND DEFINITE LEARNING}

The observed opposite learning-induced shifts toward either more or less PV/GAD67 expression and excitatory/ inhibitory synaptic puncta distributions within the ensemble of local PV neurons might reflect concerted global network shifts possibly involving most PV neurons. Alternatively, there might be PV neurons susceptible to lowPV plasticity during provisional learning, and other PV neurons susceptible to high-PV plasticity upon definite learning. Indeed, using BrdU-mediated birth-dating during medial ganglionic eminence development, we found that PV basket cells consist of two previously unrecognized subpopulations, which are specified during the first and second half of neurogenesis in mouse subpallium and differ in their connectivities and plasticity regulation (Fig. 2A-D; Donato et al. 2015). Early-born PV basket cells generated during the first half of neurogenesis show comparatively high-PV/GAD67 levels and excitation/inhibition connectivity ratios under baseline conditions, which in most cases further increase upon learning-induced plasticity (Fig. 2A-C). Furthermore, early-born PV neurons show plasticity upon $\mathrm{cFC}$ and at the end of maze learning, but not upon environmental enrichment (EE) or during maze learning (Fig. 2C). Early-born PV basket cells appear to account for characteristic features of mature fastspiking PV neuron networks such as narrow synchronization windows and learning-related $\theta-\gamma$ entrainment within and among brain systems (Bartos et al. 2007). Thus, highPV and GAD67 levels enhance fast and high-frequency firing properties of PV neuron networks important for $\gamma$ band network activity (Fuchs et al. 2007; Cardin et al. 2009; Kuhlman et al. 2010). In contrast, late-born PV basket cells specified during the second half of neurogenesis show low-PV/GAD67 levels and excitation/inhibition connectivity ratios under baseline conditions, which in most cases further decrease upon learning-induced plasticity. Furthermore, late-born PV neurons show plasticity upon EE, during maze learning and when critical periodtype plasticity is induced (Fig. 2A-C). Notably, early-born PV neuron cell plasticity is specifically regulated by excitation, whereas late-born PV neuron cell plasticity is specifically regulated by PV neuron inhibition (Donato et al. 2015). Furthermore, cell plasticity in early-born neurons mainly involved alterations in the densities of excitatory synaptic puncta, whereas cell plasticity in late-born neurons mainly involved alterations in inhibitory synaptic puncta densities onto PV neuron dendrites. These matched specificities of regulation were particularly striking in comparisons of how $\mathrm{cFC}$ or EE induced PV cell plasticity in CA3/CA1 versus DG. Thus, although cFC consistently involved early-born neuron plasticity and EE consistently involved late-born neuron plasticity, the signs of PV (and Mef2a) changes were opposite in the hippocampal subdivisions, and this was reflected in opposite signs of excitatory (and inhibitory) connectivity regulation in the two subpopulations (Donato et al. 2015).

The consistent experience-related plasticity regulation of the two subpopulations of PV basket cells raised the issue of whether these might also show distinct output targets related to behavioral function (Kepecs and Fishell 2014; Lee et al. 2014). Indeed, we found that early-born PV neurons preferentially target deep cells in the pyramidal layer of hippocampal CA1, whereas late-born PV neurons preferentially target superficial cells (Fig. 2D). The selective connectivity between early-/late-born PV neurons and early-/late-born pyramidal cells is reminiscent of selective connectivity among principal neuron subpopulations in hippocampal dentate gyrus, CA3 and CA1, suggesting that it might reflect circuit assembly principles based on relative schedules of neurogenesis and neuronal maturation (Deguchi et al. 2011). Although the functional implications of the selective output connectivity of PV neuron subpopulations remain to be determined, our findings would be consistent with the notion that the distinct excitation/inhibition input ratios onto early- and late-born PV neurons and the specific regulation upon learning 
A

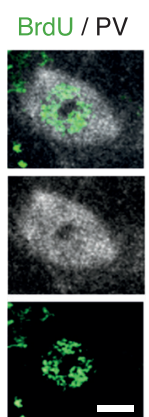

C

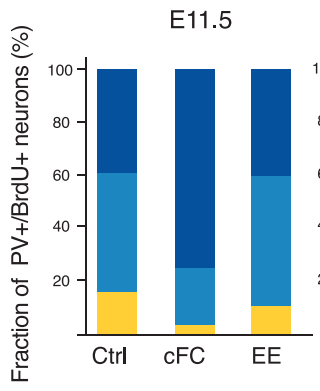

Hippocampal CA3, adult

.5 E11.5 E13.5 E15.5

BrdU injection

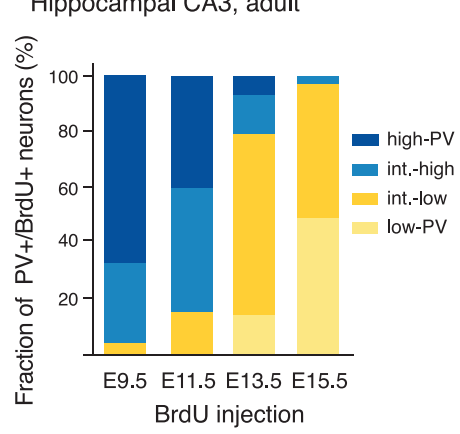

D

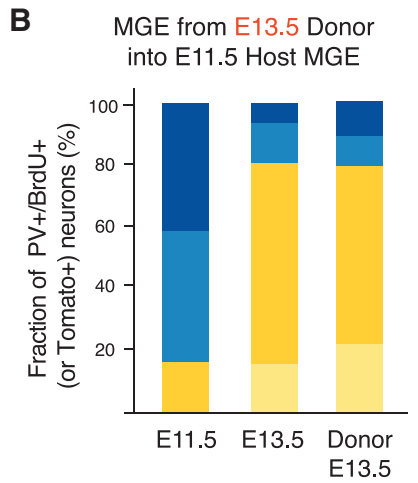

Hippocampal CA1
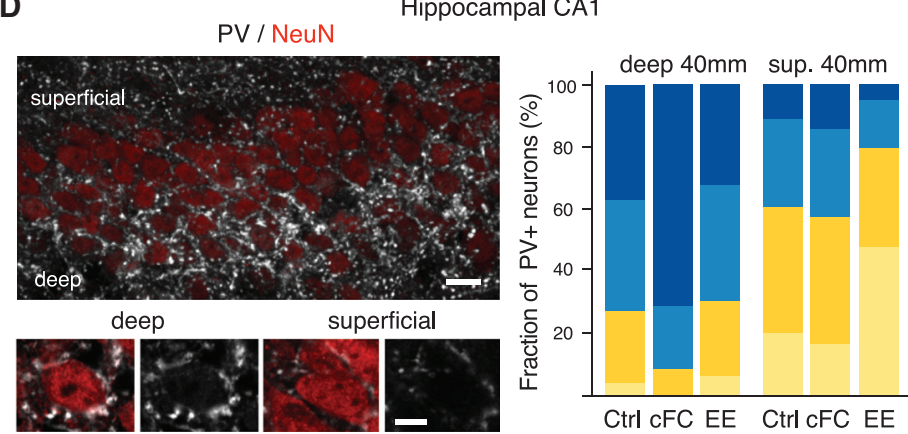

Figure 2. Developmentally specified subpopulations of PV basket cells showing plasticity upon definite or provisional learning. $(A)$ Relationship between schedule of neurogenesis and PV/GAD67 levels in adult hippocampal CA3b. (Left) Representative example of PV neuron in adult hippocampal CA3b labeled with BrdU, and scatter plot of PV labeling values for cells (individual dots) labeled at E9.5, E11.5, E13.5, and E15.5. (Right) Labeling value distributions as a function of birth date. (B) Grafting E13.5 MGE cells into E11.5 host. Adult distribution of PV labeling intensities in donor neurons closely matched to late-born PV neurons of host. $(C)$ Time schedule of neurogenesis versus regulation of PV levels upon cFC or EE. $(D)$ PV signals of perisomatic boutons at deep- and superficial-layer CA1 pyramidal cells. (Left) Representative examples of bouton signal distributions at deep- and superficial-layer pyramidal neurons. Scale bars, $15 \mu \mathrm{m}$ (top) and $5 \mu \mathrm{m}$ (bottom). (Right) PV-level distributions of perisomatic boutons at deep- and superficial-layer CA1 pyramidal cells upon cFC or EE. (Adapted from Donato et al. 2015.)

might reflect information flow through functionally distinct microcircuits (Fig. 3; Mizuseki et al. 2011; Lapray et al. 2012; Knierim et al. 2014; Cembrowski et al. 2016; Lagler et al. 2016; English et al. 2017; Soltesz and Losonczy 2018).

\section{LEARNING-INDUCED PV NEURON PLASTICITY: DELAYED ONSET AND PERSISTENCE FOR DAYS}

To investigate which aspects of learning and memory might be influenced by learning-induced plasticity in local PV neurons, we monitored the time course of high-PV plasticity induced in mouse hippocampal CA3 upon cFC. In ventral (vH) and dorsal hippocampus (dH), PV neuron labeling distributions were not detectably altered up to $+4.5 \mathrm{~h}$ (i.e., $4.5 \mathrm{~h}$ after acquisition) and had reached peak high-PV values at $+6 \mathrm{~h}$. These were maintained until $+30 \mathrm{~h}$, had decreased to half-maximal values around +36 $\mathrm{h}$, and reached again baseline values at $+48 \mathrm{~h}$ after acquisition (Fig. 4A). Low-PV plasticity induced upon $1 \mathrm{~d}$ of MWM training also only became detectable at +6 h. Notably, and like for high- or low-PV plasticity induced upon learning, pharmacogenetically induced PV plasticity only became detectable at $+6 \mathrm{~h}$ (and not yet at $+4.5 \mathrm{~h}$ ) upon induction (Karunakaran et al. 2016). Unlike plasticity induced upon learning, elevated contents of high- or low-PV neurons begun to decline from +10 to $11 \mathrm{~h}$, and had returned to baseline values at $+15 \mathrm{~h}$ (Fig. 4B). These observations suggested that long-lasting PV plasticity possibly required for long-term memory consolidation might depend on additional signals delivered locally. Given the role of late dopamine (DA) signaling in fear memory consolidation, we hypothesized that this might involve local DA signaling acting through D1/5 receptors. Indeed, local delivery of a specific $\mathrm{D} 1$ receptor agonist to $\mathrm{vH}$ at $+12 \mathrm{~h}$ rescued and extended both the high- and the low-PV shifts up to at least $+24 \mathrm{~h}$ (Fig. 4B; Karunakaran et al. 2016). Notably, local delivery of D1 agonist to $\mathrm{vH}$ of naive mice induced dramatic increases in the fraction of pDARPP-32+ and pERK+ PV neurons, whereas corresponding changes in non-PV neurons were much more modest (pDARPP-32) or absent (pERK), suggesting that D1R might signal in PV neurons to modulate PV neuron plasticity (Karunakaran et al. 2016).

Given the dependence on D1R signaling by the PV neuron plasticity induced upon pharmacogenetic activation or inhibition of PV neurons, we hypothesized that PV neuron plasticity induced upon learning might be sustained through endogenous DA signaling onto D1R. Indeed, local delivery of D1R antagonist any time between 
A

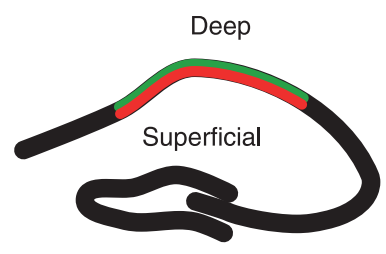

B



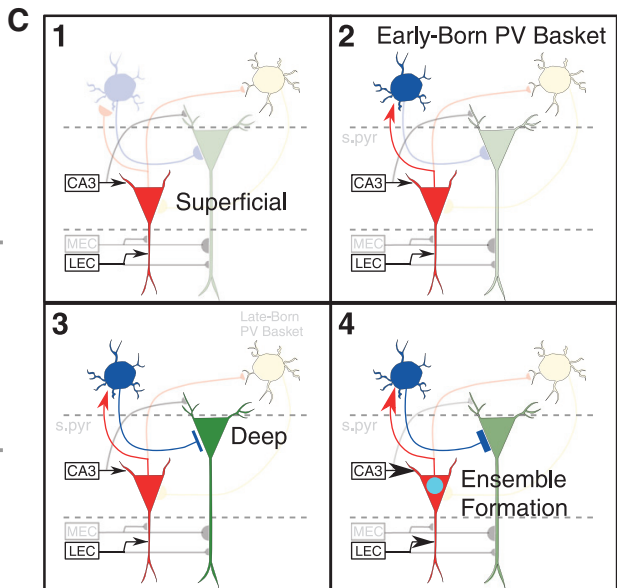

Figure 3. Distinct local microcircuit connectivities for early- and late-born PV neurons in hippocampal CA1. $(A)$ Position of deep (green) and superficial (red) pyramidal neurons in CA1. (B) Schematic representation of connectivity involving deep/superficial and early-/late-born PV neurons. (C) Proposed sequence of recruitments (1-4) for a hypothetical superficial (1) to early-born PV neuron (2) to deep pyramidal neuron (3) local circuit. Panel 4 indicates how the microcircuit might support the formation of neuronal ensembles in superficial pyramidal neurons.

+10 and $+16 \mathrm{~h}$ after $\mathrm{cFC}$ or water maze learning led to a complete loss of any detectable local PV plasticity within $10-15$ min. Furthermore, local delivery of D1R antagonist to $\mathrm{vH}$ at $+12 \mathrm{~h}$ upon $\mathrm{cFC}$ suppressed any subsequent freezing to context. This finding led us to determine when local delivery of D1R antagonist to $\mathrm{vH}$ influences freezing to context upon $\mathrm{cFC}$. These experiments revealed the existence of two sensitive time windows to influence consolidation of learning, one up to $+5-6 \mathrm{~h}$ after acquisition, and the second one between +10 and $+15 \mathrm{~h}$ (Karunakaran et al. 2016). The first time window was closely correlated to the delay period preceding the appearance of detectable PV neuron plasticity, whereas the second one was reminiscent of a previously described time window involving immediate early gene expression and offline network activity essential for long-term memory consolidation (Fig. 4C). The unusual features of delayed onset, and prolonged persistence of the PV plasticity raise the question of what might be its roles in learning and memory.

\section{SIX-HOUR TIME WINDOW UPON ACQUISITION TO DEFINE WHAT WILL BE CONSOLIDATED}

The 5- to 6-h time window after learning, when PV plasticity is not yet detectable, coincides with a time when memories of related experiences are merged into shared neuronal assemblies (Cai et al. 2016; Rashid et al. 2016). As recently reported, this corresponds to a time window when information acquired separately, but linked through shared elements such as context or taskrelevant objects, is combined to determine whether and what is being learned (time units for learning) (Chowdhury and Caroni 2018). The duration of the time units depends on cFos protein expression and function in local neuronal assemblies, which becomes detectable $45 \mathrm{~min}$ after acquisition, and is maintained against proteasomemediated degradation during about $4-5 \mathrm{~h}$ through processes depending on offline network activity within brain systems involved in that particular learning (Chowdhury
A

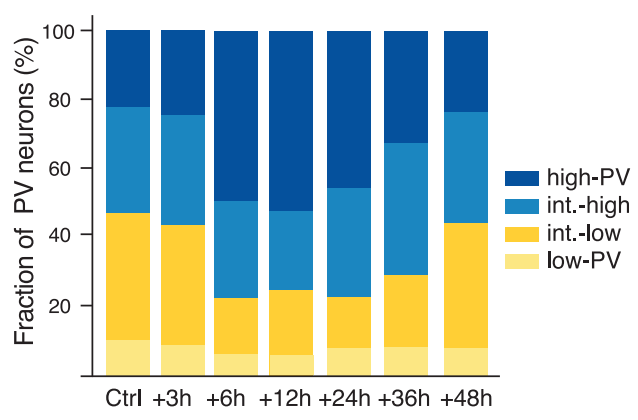

B Pharmacogenetic PV activation

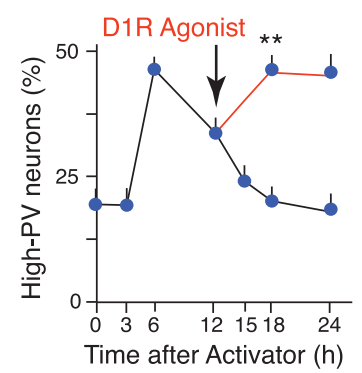

C

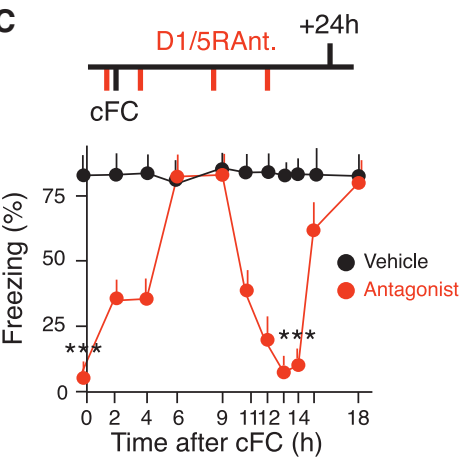

Figure 4. Regulation of PV neuron plasticity by D1R signaling. (A) Time course of high-PV neuron plasticity induced upon cFC in $\mathrm{vH}$ CA3. (B) Time course of high-PV neuron plasticity induced upon pharmacogenetic activation of PV neurons in CA3 and prolongation of the plasticity upon local delivery of D1R agonist. $(C)$ Two time windows after acquisition of cFC when local delivery of D1/5R antagonist to $\mathrm{vH}$ interferes with long-term consolidation of fear memory (tested at $+24 \mathrm{~h}$ ). (Adapted in part from Karunakaran et al. 2016.) 
A
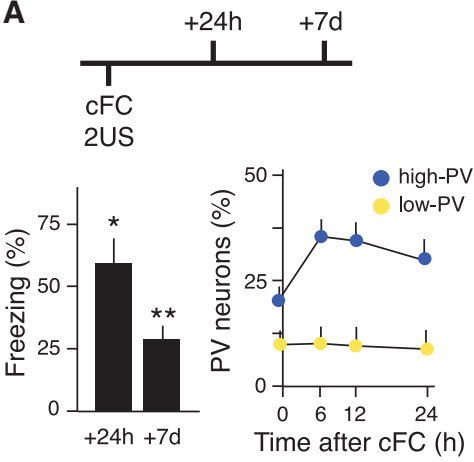

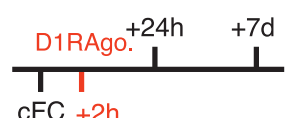

$\mathrm{FC}+2 \mathrm{~h}$ 2US



B

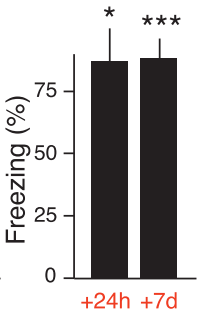

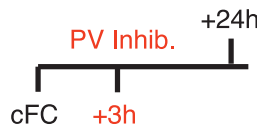

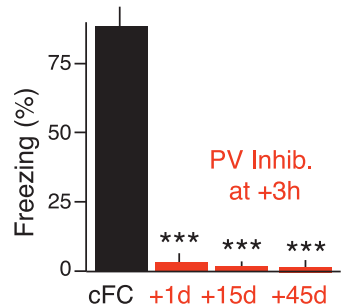

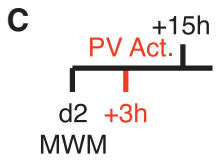

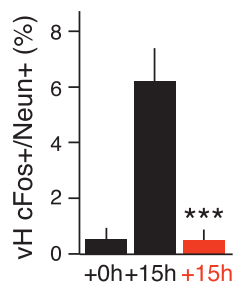

Figure 5. Modulation of PV plasticity and long-term memory during early time window up to $5-6 \mathrm{~h}$ after acquisition of cFC. $(A)$ Strengthening of weak 2US PV plasticity and fear memory (left) upon delivery of D1R agonist to vH at $+2 \mathrm{~h}($ right). (B) Suppression of long-term fear memory upon local delivery of $\mathrm{D} 1 / 5 \mathrm{R}$ antagonist to $\mathrm{vH}$ at $+3 \mathrm{~h}$ after acquisition of cFC. Control bar: test at $+24 \mathrm{~h}$. $(C)$ Suppression of $\mathrm{vH}$ cFos induction at $+15 \mathrm{~h}$ induced upon water maze learning by local induction of opposite-sign PV neuron plasticity (high-PV) in $\mathrm{vH}$ at $+3 \mathrm{~h}$. (Adapted from Karunakaran et al. 2016.)

and Caroni 2018). The 5-6 h time window that precedes detectable PV plasticity might therefore reflect a time when the magnitude, and possibly also the sign (toward high- vs. low-PV), of the PV neuron plasticity might be influenced by experience.

To determine whether the status of D1/5 receptor activation up to $5 \mathrm{~h}$ after acquisition might modulate the strength of PV plasticity and learning, we performed experiments in mice that underwent a comparatively weak $\mathrm{cFC}$ protocol. The unconditioned stimulus (US) (here foot shock) is indicated in the following as US. A $2 \times$ US (instead of $5 \times$ US) protocol resulted in a comparatively modest high-PV shift in $\mathrm{vH}$ at +12 and $+24 \mathrm{~h}$ after acquisition. In parallel, when compared to a $5 \times \mathrm{US}$ protocol, the $2 \times \mathrm{US}$ protocol produced a reduced freezing response at $+24 \mathrm{~h}$, and strongly reduced freezing at $+7 \mathrm{~d}$. Local application of D1 receptor agonist to $\mathrm{vH}$ at $+2 \mathrm{~h}$ after acquisition of $2 \times \mathrm{US}$ fear conditioning produced a sustained enhancement of $\mathrm{vH}$ high-PV contents, and freezing to context at $+24 \mathrm{~h}$ and at $+7 \mathrm{~d}$ was now undistinguishable to fear memory upon a $5 \times$ US protocol (Fig. 5A; Karunakaran et al. 2016). These and related results provided evidence that D1/5 receptor signaling during the $+0-5 \mathrm{~h}$ time window after acquisition modulates the strength of subsequent PV plasticity and fear memories.

In a second approach, we pharmacogenetically imposed a low-PV shift (i.e., plasticity of opposite sign to that induced by learning) at $+3 \mathrm{~h}$ after cFC specifically in vH PV neurons. Although memory recalled at $+6.5 \mathrm{~h}$ was not affected, the procedure led to suppression of freezing to context (i.e., of any behaviorally detectable memory) at $+24 \mathrm{~h}$, and up to at least $7 \mathrm{wk}$. Likewise, when high-PV plasticity was imposed pharmacogenetically at $+3 \mathrm{~h}$ after water maze learning (a learning processes inducing lowPV plasticity), no learning-related cFos expression was detected at $+15 \mathrm{~h}$, and no learning was detected behaviorally on the subsequent day (Fig. 5B,C).

Therefore, although alterations in PV levels only become detectable $6 \mathrm{~h}$ after acquisition, PV plasticity is already modulated during the first $5 \mathrm{~h}$ after acquisition through endogenous D1/5 receptor signaling, providing a potential mechanism through which events occurring subsequent to the initial acquisition process might influence long-term memory consolidation processes at $+12-$ $14 \mathrm{~h}$. Notably, interventions that specifically suppressed detectable learning-induced PV plasticity from $+6 \mathrm{~h}$ on did not affect intermediate memory tested, for example, at $+6.5 \mathrm{~h}$. These results suggest that requirements for consolidation of short- and intermediate-term memories differ from those for long-term memories.

\section{TWELVE-HOUR TIME WINDOW FOR PV PLASTICITY-DEPENDENT MEMORY CONSOLIDATION}

In good agreement with reports that D1R signaling $12 \mathrm{~h}$ after acquisition is important for long-term consolidation and strengthening of fear memories, we found that PV neuron plasticity sustained during a $+11-15 \mathrm{~h}$ time window is critically important for enhanced ripple densities, for the cFos peak at $+15 \mathrm{~h}$, and for long-term memory consolidation, causally tying the PV plasticity to the occurrence of key offline network and cell assembly processes involved in memory consolidation (Fig. 6; Karunakaran et al. 2016). We further found that local D1/5 receptor signaling at $+12-14 \mathrm{~h}$ is specifically required in PV neurons to ensure maintenance of learning-induced PV plasticity critically important for long-term memory consolidation (Fig. 6A). Incidental learning (e.g., object recognition) not involving reinforcement but involving a second peak of cFos expression at $+12 \mathrm{~h}$ did not show PV neuron plasticity and did not depend on D1R signaling at $+12-14$ h for long-term memory consolidation, suggesting that a requirement for local D1/5 receptor signaling at $+12-14 \mathrm{~h}$ is a shared feature of reinforced learning involving PV plasticity.

Mechanistically, PV basket cell recruitment enhances network activity through synchronization of principal neurons to support coordinated fast network activities such as ripples, spindles, and $\gamma$-range oscillations (Stark et al. 2014), which are critically important for long-term memory consolidation (Buzsáki 2015). The circuit mechanisms through which opposite-sign low- and high-PV 
A

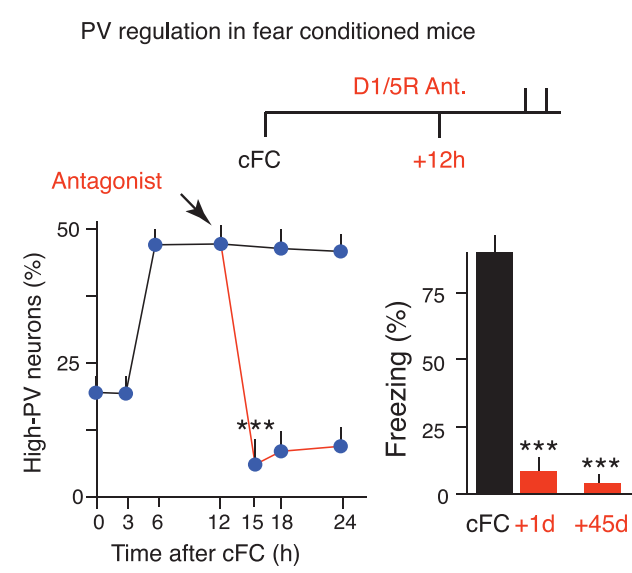

D
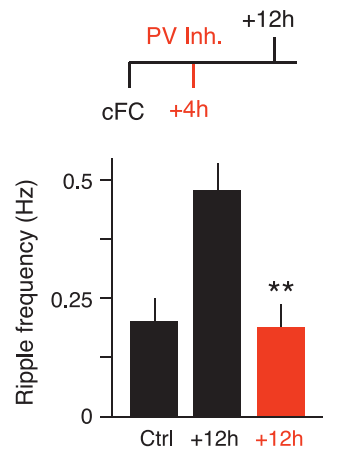
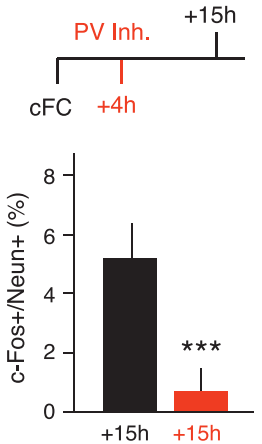

B





cFC: $\mathrm{vH}$ sharp wave ripples
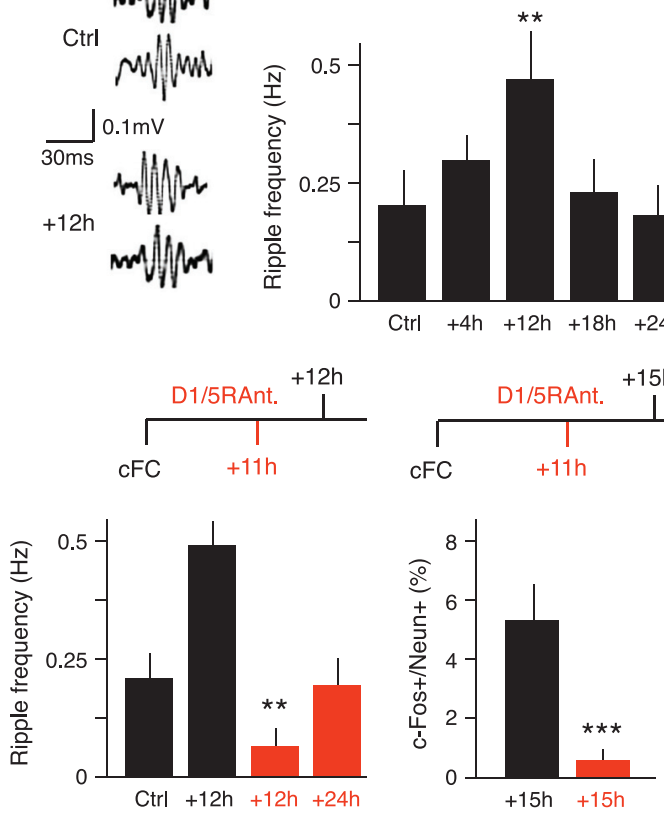

Figure 6. Modulation of PV plasticity, ripple activity, cFos expression, and long-term memory during late time window at $+11-15 \mathrm{~h}$ after acquisition of cFC. (A) Rapid loss of high-PV neuron plasticity and loss of fear memory upon local delivery of D1/5R antagonist to $\mathrm{vH}$ $12 \mathrm{~h}$ after $\mathrm{cFC}$. PV neuron analysis in $\mathrm{vH}$ at different time points upon $\mathrm{cFC}$. $(B, C)$ Time course of cFos expression $(B)$ and sharp wave ripple induction $(C)$ in $\mathrm{vH}$ upon $\mathrm{cFC}$. $(D)$ Enhanced ripple density at $+12 \mathrm{~h}$ after cFC and cFos expression at $+15 \mathrm{~h}$ after $\mathrm{cFC}$ are suppressed upon pharmacogenetic induction of low-PV plasticity (i.e., opposite sign to $\mathrm{cFC}$ ) at $+4 \mathrm{~h}$ (two left panels) or local delivery of $\mathrm{D} 1 / 5 \mathrm{R}$ antagonist at $+11 \mathrm{~h}$ (two right panels). All treatments and analyses were performed in $\mathrm{vH}$. (Adapted from Karunakaran et al. 2016.)

plasticity specifically ensure long-term consolidation of provisional versus definite memories remain to be determined, but one plausible hypothesis involves distinct local networks and related neuronal assemblies in learning involving low- or high-PV plasticity (Fig. 3).

Why might long-term memory consolidation depend on organized network activity and cellular plasticity induced about $12 \mathrm{~h}$ after acquisition, long after known biochemical and cellular processes involved in memory consolidation (Redondo and Morris 2011) have subsided? It is possible that slow biochemical processes affecting synaptic rearrangement processes involved in memory might require such a long time, but such a process would seem to put long-term memories at risk of interference through unrelated network activity. A further possibility could have been that the late time window might coincide with network processes during sleep, a brain state known to have an important role in memory consolidation, but direct testing of this possibility suggested that the time window occurred at the same delay from the original learning process, irrespective of the time of the day or night at which it had occurred (Karunakaran et al. 2016). Instead, we propose that the late time window might serve to separate learning processes occurring on any given day from the long-lasting consequences of the learning for the animal's behavior (which includes integration with previous learning and memory processes). Such a view is consistent with our recent findings that the essential role of infralimbic cortex in subsequent alternative learning (e.g., fear extinction learning) is set up during $\mathrm{a}+11-14 \mathrm{~h}$ time window after fear learning through circuit and systems interactions involving specific reciprocal connectivity between prelimbic and infralimbic cortex (Mukherjee and Caroni 2018). 
A


B
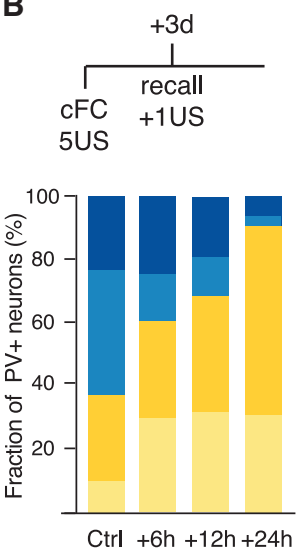

C
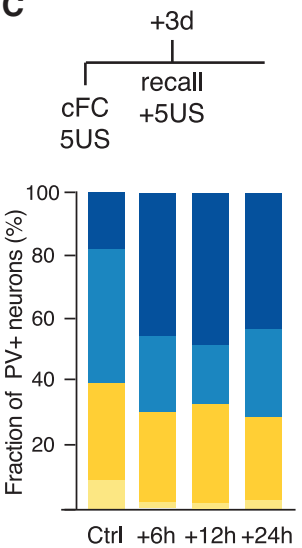

D
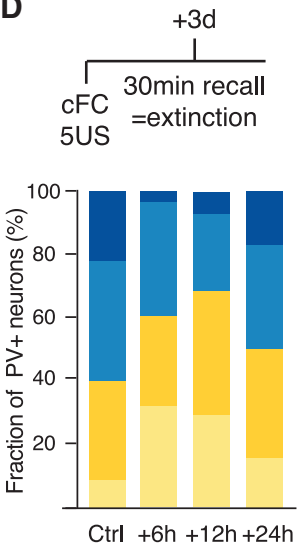

E

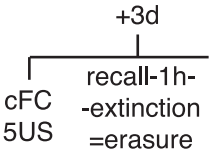

Figure 7. Dynamics of $\mathrm{dH}$ PV plasticity upon recall of cFC. All data: recall $3 \mathrm{~d}$ after $5 \mathrm{US} \mathrm{cFC}$; analysis in CA3b. $(A-E)$ Analysis at +6 , +12 , and $+24 \mathrm{~h}$ after recall $(A)$, recall $+1 \mathrm{US}(B)$, recall $+5 \mathrm{US}(C), 30$-min recall (extinction) $(D)$, and 5 -min recall followed $1 \mathrm{~h}$ later by 30 -min recall (erasure) $(E)$.

Additional findings from our laboratory focusing on the dynamics of PV neuron plasticity in area CA3 of $\mathrm{dH}$ upon recall of fear memory under different experimental conditions provide further support for a role of the $+11-14 \mathrm{~h}$ long-term consolidation window in consolidating behaviorally adaptive memories. Recall of fear memory (i.e., exposure to conditioned context in the absence of foot shocks) $3 \mathrm{~d}$ after cFC induced low-PV plasticity at $+6 \mathrm{~h}$, which changed to baseline-like values at $+12 \mathrm{~h}$, and to high-PV plasticity at $+24 \mathrm{~h}$ (Fig. 7A). Interfering with the PV plasticity at $+12 \mathrm{~h}$ prevented detectable high-PV plasticity at $+24 \mathrm{~h}$ and produced a permanent loss of the fear memory, suggesting that the late shift to high-PV plasticity was necessary for reconsolidation of the fear memory. When recall was paired to 1 US instead of the 5US during fear conditioning, the low-PV plasticity persisted through the 12 -h time window and had become more robust at $+24 \mathrm{~h}$ (Fig. 7B). In contrast, if recall was paired to 5US, high-PV plasticity was induced at $+6 \mathrm{~h}$, and the plasticity lasted at least until $+24 \mathrm{~h}$ (Fig. 7C). These and additional findings suggest that experience deviating from expectations (no US or only 1 US instead of 5US) initially induces low-PV plasticity, which is followed by a reassessment of the new learning to either reconsolidate the original fear learning (no US), or to prepare for possible further learning (1US). In further related experiments, and extinction protocol (30 min of recall in the absence of US) induced low-PV plasticity at $+6 \mathrm{~h}$, which persisted through the +12 -h time window (Fig. 7D). In stark contrast, an erasure protocol (5 min recall followed $1 \mathrm{~h}$ later by a 30 -min extinction protocol) induced high-PV plasticity at $+6 \mathrm{~h}$, which persisted up to and beyond $+24 \mathrm{~h}$ (Fig. 7E). Within the context of learningrelated PV plasticity, extinction represents a transient adjustment to absence of US (i.e., provisional learning), whereas erasure leads to a permanent loss of the association between context and fear (i.e., definite learning).

Taken together, these findings provide evidence that $\mathrm{PV}$ neuron plasticity induced upon experience, and detected at $+6 \mathrm{~h}$, can be subsequently modified without further behavioral experience during the $+11-15$-h time window of long-term memory consolidation, leading to PV neuron plasticity that reflects the long-term behavioral consequences of the experience. With other words, and consistent with our hypothesis, the late time window for memory consolidation appears to reflect a time when local plasticity is readjusted through offline network activity to match the corresponding long-term memory.

\section{CONCLUSION}

PV basket cells, the most abundant local inhibitory interneurons in the brain, show dramatic cellular and structural synaptic plasticity upon reinforced learning. The plasticity comes in two flavors: one ("high-PV") that leads to increased expression of PV neuron functional markers such as PV and GAD67 (Volman et al. 2011; Lazarus et al. 2015) and increased densities of excitatory synaptic puncta onto PV neurons, and one ("low-PV") that leads to decreased expression of PV/GAD67 and increased densities of inhibitory synaptic puncta onto PV neurons. High-PV plasticity is implemented by early-born PV neurons, whose plasticity is induced by changes in PV neuron excitation, whereas low-PV plasticity is implemented by late-born PV neurons, whose plasticity is induced by changes in PV neuron inhibition. Low-PV plasticity is specifically induced upon provisional reinforced learning, whereas high-PV plasticity is specifically induced upon definite reinforced learning. PV neuron plasticity becomes detectable at the end of time units for learning, once experience and signaling collected during 5-6 h upon initial acquisition determine whether and what will be learned. Notably, the PV plasticity is critically important for longterm consolidation of reinforced memories during a time window $+11-15 \mathrm{~h}$ after acquisition. The mechanisms through which PV plasticity ensure long-term memory consolidation appear to involve support of offline network activity specifically required for enhanced ripple activity 
and immediate early gene expression in neuronal assemblies thought to encode the corresponding memories (Singer and Frank 2009; van de Ven et al. 2016; Ognjanovski et al. 2017; Rothschild et al. 2017; Xia et al. 2017). Longterm consolidation of nonreinforced (incidental) memories also depends on a time window around $+12 \mathrm{~h}$, when cFos in corresponding assemblies needs to be re-expressed, but the memories do not depend on local D1R signaling and PV neuron plasticity for long-term consolidation. These findings suggest the existence of fundamental differences between reinforced and nonreinforced memories and their consolidation, possibly reflecting the more consequential impact of reinforced memories for behavior.

Enhanced functionality of early-born PV neuron networks upon definite learning might promote consolidation of strong memories within and between brain systems. Such a mechanism might account for the enhanced $\gamma$-phase coupling between entorhinal cortex and hippocampal CA1 that was detected during late phases of hippocampal learning (Igarashi et al. 2014). Selective connectivity to and from specific functional subpopulations of principal neurons might ensure specific stabilization of neuronal assemblies encoding definite learning memories. Likewise, reduced functionality of late-born PV neuron networks might be permissive to bind assemblies of more flexible and possibly less strongly interconnected neuronal assemblies. This form of plasticity appears to be the counterpart of critical period plasticity and of how EE supports learning and brain function (Pizzorusso et al. 2002). Like for definite learning assemblies, provisional memory assemblies likely involve dedicated subpopulations of principal neurons and specific connectivity to and from early-born PV neurons.

These findings raise a number of questions for further research. One of them involves the likely prospect that provisional and definite learning might be implemented through partially separate circuits within and possibly also between brain areas. Identifying the subpopulations of neurons and the connectivity principles involved in such functional subcircuits will likely provide fundamental insights into how the brain deals with coexisting variability and stability in learning and memory (Grosmark and Buzsáki 2016; Clopath et al. 2017). A second important question involves the systems mechanisms for memory consolidation during the late time window $(+11-15 \mathrm{~h})$ after acquisition. Elucidating principles of how previous knowledge might be integrated with recent memories during offline network activity to support adaptive learning and behavior will not only provide important advances into mechanisms of learning and memory, but might also provide valuable entry points to begin to investigate mechanisms of long-term systems consolidation in the brain. Finally, it might be useful to consider the possibility that long-term cellular plasticity involved in learning and memory might not be exclusive to PV basket cells.

\section{ACKNOWLEDGMENTS}

The authors gratefully acknowledge the many people who have contributed to these projects over the years in our laboratory. This research was supported by SYNAPSY, a National Center of Competence Research funded by the Swiss National Science Foundation, by the European Research Center (MemoryDynamics), and by the Friedrich Miescher Institute for Biomedical Research.

\section{REFERENCES}

Bartos M, Vida I, Jonas P. 2007. Synaptic mechanisms of synchronized $\gamma$ oscillations in inhibitory interneuron networks. Nat Rev Neurosci 8: 45-56. doi:10.1038/nrn2044

Bekinschtein P, Cammarota M, Igaz L, Bevilaqua LRM, Izquiero I, Medina JH. 2007. Persistence of long-term memory storage requires a late protein synthesis- and BDNF-dependent phase in the hippocampus. Neuron 53: 261-277. doi:10.1016/j .neuron.2006.11.025

Buzsáki G. 2015. Hippocampal sharp wave-ripples: a cognitive biomarker for episodic memory and planning. Hippocampus 25: 1073-1188. doi:10.1002/hipo.22488

Buzsáki G, Wang XJ. 2012. Mechanisms of $\gamma$ oscillations. Annu Rev Neurosci 35: 203-225. doi:10.1146/annurev-neuro062111-150444

Cai DJ, Aharoni D, Shuman T, Shobe J, Biane J, Song W, Wei B, Veshkini M, La-Vu M, Lou J, et al. 2016. A shared neural ensemble links distinct contextual memories encoded close in time. Nature 534: 115-118. doi:10.1038/nature 17955

Cardin JA, Carlen M, Meletis K, Knoblich U, Zhang F, Deisseroth K, Tsai LH, Moore CI. 2009. Driving fast-spiking cells induces $\gamma$ rhythm and controls sensory responses. Nature 459: 663-667. doi:10.1038/nature08002

Caroni P, Chowdhury A, Lahr M. 2014. Synapse rearrangements upon learning: from divergent-sparse connectivity to dedicated sub-circuits. Trends Neurosci 37: 604-614. doi:10.1016/j tins.2014.08.011

Carr MF, Jadhav SP, Frank LM. 2011. Hippocampal replay in the awake state: a potential substrate for memory consolidation and retrieval. Nat Neurosci 14: 147-153. doi:10.1038/nn .2732

Cembrowski MS, Bachman JL, Wang L, Sugino K, Shields BC, Spruston N. 2016. Spatial gene-expression gradients underlie prominent heterogeneity of CA1 pyramidal neurons. Neuron 89: 351-368. doi:10.1016/j.neuron.2015.12.013

Chowdhury A, Caroni P. 2018. Time units for learning involving maintenance of system-wide cFos expression in neuronal assemblies. Nat Commun 9: 4122. doi:10.1038/s41467-01806516-3

Clopath C, Bonhoeffer T, Hübener M, Rose T. 2017. Variance and invariance of neuronal long-term representations. Philos Trans R Soc Lond B Biol Sci 372: 20160161. doi:10.1098/rstb .2016 .0161

Deguchi Y, Donato F, Galimberti I, Cabuy E, Caroni P. 2011. Temporally matched subpopulations of selectively interconnected principal neurons in the hippocampus. Nat Neurosci 14: 495-504. doi:10.1038/nn.2768

Di Cristo G, Chattopadhyaya B, Kuhlman SJ, Fu Y, Bélanger MC, Wu CZ, Rutishauser U, Maffei L, Huang J. 2007. Activity-dependent PSA expression regulates inhibitory maturation and onset of critical period plasticity. Nat Neurosci 10: 15691577. doi:10.1038/nn2008

Donato F, Rompani S, Caroni P. 2013. Parvalbumin-expressing basket-cell network plasticity induced by experience regulates adult learning. Nature 504: 272-276. doi:10.1038/ nature 12866

Donato F, Chowdhury A, Lahr M, Caroni P. 2015. Early- and lateborn Parvalbumin basket cell subpopulations exhibiting distinct regulation and roles in learning. Neuron 85: 770-786. doi:10.1016/j.neuron.2015.01.011

English DF, McKenzie S, Evans T, Kim K, Yoon E, Buzsáki G. 2017. Pyramidal cell-interneuron circuit architecture and dynamics in hippocampal networks. Neuron 96: 505-520.e7. doi:10.1016/j.neuron.2017.09.033 
Fuchs EC, Zivkovic AR, Cunningham MO, Middleton S, Lebeau FE, Bannerman DM, Rozov A, Whittington MA, Traub RD, Rawlins JN, et al. 2007. Recruitment of parvalbumin-positive interneurons determines hippocampal function and associated behavior. Neuron 53: 591-604. doi:10.1016/j.neuron.2007.01 .031

Girardeau G, Benchenane K, Wiener SI, Buzsáki G, Zugaro MB. 2009. Selective suppression of hippocampal ripples impairs spatial memory. Nat Neurosci 12: 1222-1223. doi:10.1038/ nn. 2384

Girardeau G, Cei A, Zugaro M. 2014. Learning-induced plasticity regulates hippocampal sharp wave-ripple drive. $J \mathrm{Neu}$ rosci 34: 5176-5183. doi:10.1523/JNEUROSCI.4288-13 .2014

Grosmark A, Buzsáki G. 2016. Diversity in neural firing dynamics supports both rigid and learned hippocampal sequences. Science 351: 1440-1443. doi:10.1126/science.aad1935

Hensch TK. 2005. Critical period plasticity in local cortical circuits. Nat Rev Neurosci 6: 877-888. doi:10.1038/nrn1787

Hensch T, Fagiolini M, Mataga N, Stryker MP, Baekkeskov S, Kash SF. 1998. Local GABA circuit control of experiencedependent plasticity in developing visual cortex. Science 282: 1504-1508. doi:10.1126/science.282.5393.1504

Hu H, Gan J, Jonas P. 2014. Interneurons. Fast-spiking, parvalbumin $^{+}$GABAergic interneurons: from cellular design to microcircuit function. Science 345: 1255263. doi:10.1126/ science. 1255263

Igarashi KM, Lu L, Colgin LL, Moser MB, Moser EI. 2014. Coordination of entorhinal-hippocampal ensemble activity during associative learning. Nature 510: 143-147. doi:10 .1038 /nature 13162

Isaacson JS, Scanziani M. 2011. How inhibition shapes cortical activity. Neuron 72: 231-243. doi:10.1016/j.neuron.2011.09 .027

Jadhav SP, Kemere C, German PW, Frank LM. 2012. Awake hippocampal sharp-wave ripples support spatial memory. Science 336: 1454-1458. doi:10.1126/science.1217230

Kaelbling LP, Littman ML, Moore AW. 1996. Reinforcement learning: a survey. J Artif Intell Res 4: 237-285. doi:10 .1613/jair.301

Karunakaran S, Chowdhury A, Donato F, Quairiaux C, Michel C, Caroni P. 2016. PV plasticity sustained through D1/5 dopamine signaling required for long-term memory consolidation. Nat Neurosci 19: 454-464. doi:10.1038/nn.4231

Katche C, Bekinschtein P, Slipczuk L, Goldin A, Izquierdo I, Cammarota M, Medina JH. 2010. Delayed wave of c-Fos expression in the dorsal hippocampus involved specifically in persistence of long-term memory storage. Proc Natl Acad Sci 107: 349-354. doi:10.1073/pnas.0912931107

Katche C, Cammarota M, Medina JH. 2013. Molecular signatures and mechanisms of long-lasting memory consolidation and storage. Neurobiol Learn Mem 106: 40-47. doi:10.1016/j .nlm.2013.06.018

Kepecs A, Fishell G. 2014. Interneuron cell types are fit to function. Nature 505: 318-326. doi:10.1038/nature12983

Knierim JJ, Neunuebel JP, Deshmukh SS. 2014. Functional correlates of the lateral and medial entorhinal cortex: objects, path integration and local-global reference frames. Philos Trans $R$ Soc Lond B Biol Sci 369: 20130369. doi:10.1098/rstb.2013 .0369

Kuhlman SJ, Lu J, Lazarus MS, Huang ZJ. 2010. Maturation of GABAergic inhibition promotes strengthening of temporally coherent inputs among convergent pathways. PLoS Comput Biol 6: e1000797. doi:10.1371/journal.pcbi.1000797

Kuhlman SJ, Olivas ND, Tring E, Ikrar T, Xu X, Trachtenberg JT. 2013. A disinhibitory microcircuit initiates critical-period plasticity in the visual cortex. Nature 501: 543-546. doi:10 .1038 /nature 12485

Lagler M, Ozdemir A, Lagoun S, Malagon-Vina H, Borhegyi Z, Hauer R, Jelem A, Klausberger T. 2016. Divisions of identified parvalbumin-expressing basket cells during working memory-guided decision making. Neuron 91: 1390-1401. doi:10.1016/j.neuron.2016.08.010
Lapray D, Lasztoczi B, Lagler M, Viney TJ, Katona L, Valenti O, Hartwich K, Borhegyi Z, Somogyi P, Klausberger T. 2012. Behavior-dependent specialization of identified hippocampal interneurons. Nat Neurosci 15: 1265-1271. doi:10.1038/nn .3176

Lazarus MS, Krishnan K, Huang ZJ. 2015. GAD67 deficiency in parvalbumin interneurons produces deficits in inhibitory transmission and network disinhibition in mouse prefrontal cortex. Cereb Cortex 25: 1290-1296. doi:10.1093/cercor/ bht 322

Lee SH, Kwan AC, Zhang S, Phoumthipphavong V, Flannery JG, Masmanidis SC, Taniguchi H, Huang ZJ, Zhang F, Boyden ES, et al. 2012. Activation of specific interneurons improves V1 feature selectivity and visual perception. Nature 488: 379383. doi: $10.1038 /$ nature 11312

Lee SH, Marchionni I, Bezaire M, Varga C, Danielson N, LovettBarron M, Soltesz I. 2014. Parvalbumin-positive basket cells differentiate among hippocampal pyramidal cells. Neuron 82: 1129-1144. doi:10.1016/j.neuron.2014.03.034

McNamara CG, Tejero-Cantero Á, Trouche S, Campo-Urriza N, Dupret D. 2014. Dopaminergic neurons promote hippocampal reactivation and spatial memory persistence. Nat Neurosci 17: 1658-1660. doi:10.1038/nn.3843

Mizuseki K, Diba K, Pastalkova E, Buzsáki G. 2011. Hippocampal CA1 pyramidal cells form functionally distinct sublayers. Nat Neurosci 14: 1174-1181. doi:10.1038/nn.2894

Mukherjee A, Caroni P. 2018. Infralimbic cortex is required for learning alternatives to prelimbic promoted associations through reciprocal connectivity. Nat Commun 9: 2727. doi: 10.1038/s41467-018-05318-x

Ognjanovski N, Schaeffer S, Wu J, Mofakham S, Maruyama D, Zochowski M, Aton SJ. 2017. Parvalbumin-expressing interneurons coordinate hippocampal network dynamics required for memory consolidation. Nat Commun 8: 15039. doi:10 .1038/ncomms15039

Pizzorusso T, Medini P, Berardi N, Chierzi S, Fawcett JW, Maffei L. 2002. Reactivation of ocular dominance plasticity in the adult visual cortex. Science 298: 1248-1251. doi:10.1126/sci ence. 1072699

Rashid AJ, Yan C, Mercaldo V, Hsiang HL, Park S, Cole CJ, De Cristofaro A, Yu J, Ramakrishnan C, Lee SY, et al. 2016. Competition between engrams influences fear memory formation and recall. Science 353: 383-387. doi:10.1126/science .aaf0594

Redondo RL, Morris RG. 2011. Making memories last: the synaptic tagging and capture hypothesis. Nat Rev Neurosci 12: 17-30. doi:10.1038/nrn2963

Rossato JI, Bevilaqua LR, Izquierdo I, Medina JH, Cammarota M. 2009. Dopamine controls persistence of long-term memory storage. Science 325: 1017-1020. doi:10.1126/science .1172545

Rothschild G, Eban E, Frank LM. 2017. A cortical-hippocampal-cortical loop of information processing during memory consolidation. Nat Neurosci 20: 251-259. doi:10.1038/nn .4457

Singer AC, Frank LM. 2009. Rewarded outcomes enhance reactivation of experience in the hippocampus. Neuron 64: 910 921. doi:10.1016/j.neuron.2009.11.016

Sohal V, Zhang F, Yizhar O, Deisseroth K. 2009. Parvalbumin neurons and $\gamma$ rhythms enhance cortical circuit performance. Nature 459: 698-702. doi:10.1038/nature07991

Soltesz I, Losonczy A. 2018. CA1 pyramidal cell diversity enabling parallel information processing in the hippocampus. Nat Neurosci 21: 484-493. doi:10.1038/s41593018-0118-0

Southwell D, Froemke RC, Alvarez-Buylla A, Stryker MP, Gandhi SP. 2010. Cortical plasticity induced by inhibitory neuron transplantation. Science 327: 1145-1148. doi:10.1126/science .1183962

Stark E, Roux L, Eichler R, Senzai Y, Royer S, Buzsáki G. 2014. Pyramidal cell-interneuron interactions underlie hippocampal ripple oscillations. Neuron 83: 467-480. doi:10.1016/j.neuron .2014 .06 .023 
Trifilieff P, Herry C, Vanhoutte P, Caboche J, Desmedt A, Riedel G, Mons N, Micheau J. 2006. Foreground contextual fear memory consolidation requires two independent phases of hippocampal ERK/CREB activation. Learn Mem 13: 349358. doi:10.1101/lm.80206

Trifilieff P, Calandreau L, Herry C, Mons N, Micheau J. 2007. Biphasic ERK1/2 activation in both the hippocampus and amygdala may reveal a system consolidation of contextual fear memory. Neurobiol Learn Mem 88: 424-434. doi:10 $.1016 /$ j.nlm.2007.05.004

van de Ven GM, Trouche S, McNamara CG, Allen K, Dupret D. 2016. Hippocampal offline reactivation consolidates recently formed cell assembly patterns during sharp waveripples. Neuron 92: 968-974. doi:10.1016/j.neuron.2016.10 .020

Volman V, Behrens MM, Sejnowski TJ. 2011. Downregulation of parvalbumin at cortical GABA synapses reduces network $\gamma$ oscillatory activity. J Neurosci 31: 18137-18148. doi:10 .1523/JNEUROSCI.3041-11.2011

Xia F, Richards BA, Tran MM, Josselyn SA, Takehara-Nishiuchi K, Frankland PW. 2017. Parvalbumin positive interneurons mediate neocortical-hippocampal interactions that are necessary for memory consolidation. eLife 6: e27868. doi:10.7554/ eLife. 27868 


\section{$\$_{\text {CSH }}^{\infty}$ Cold Spring Harbor Symposia SYMPOSIA on Quantitative Biology}

\section{Parvalbumin Interneuron Plasticity for Consolidation of Reinforced Learning}

Matteo Tripodi, Komal Bhandari, Ananya Chowdhury, et al.

Cold Spring Harb Symp Quant Biol 2018 83: 25-35 originally published online July 9, 2019 Access the most recent version at doi:10.1101/sqb.2018.83.037630

References This article cites 58 articles, 12 of which can be accessed free at: http://symposium.cshlp.org/content/83/25.full.html\#ref-list-1

Creative This article is distributed under the terms of the

Commons http://creativecommons.org/licenses/by-nc/4.0/, which permits reuse and License redistribution, except for commercial purposes, provided that the original author and source are credited.

Email Alerting Receive free email alerts when new articles cite this article - sign up in Service the box at the top right corner of the article or click here. 